\title{
Tabu Search Algorithm for Solving Waste Collection Vehicle Routing Problem
}

\author{
Siti Asnor Faraien Binti Hassan ${ }^{\mathrm{a}}$, Syarifah Zyurina Nordin ${ }^{\mathrm{b}, *}$ \\ ${ }^{a, b}$ Department of Mathematic Science, Faculty of Science, Universiti Teknologi Malaysia, 81310 UTM Johor Bahru, Johor, Malaysia. \\ *Corresponding Author: szyurina@utm.my
}

Article history :

Article history :

Received 30 Sept 2014

Accepted 13 Apr 2015

GRAPHICAL ABSTRACT

\begin{abstract}
This study considers a Waste Collection Vehicle Routing Problem where the situation happens when vehicle must make a complete trip to make disposal operation per day. The Waste Collection Vehicle Routing Problem objective is to decide the best solution where a vehicle should make the collection first between the customers since there exist larger number of customers. The method proposed to solve the Waste Collection Vehicle Routing Problem is by using Tabu Search Algorithm.
\end{abstract}

Keywords: Vehicle Routing Problem, Waste Collection, Tabu Search Algorithm

\section{INTRODUCTION}

The Vehicle Routing Problem or well known as VRP is one of the most studied combinatorial problems that is described as the problem of designing optimal delivery or collection routes from one or several depots to a number of geographically scattered cities or customers, subject to side constraints (Laporte,1991). The objective of VRP is to route the vehicles which is one route per vehicle, starting and ending at the depot, so that all customers are supplied with their demands and the total travel distance is minimized. The VRP is important in the fields of physical distribution and logistics. The distribution of goods concerns the service, in a given time period, a set of customers by a set of vehicles, which are located in one or more depots, are operated by a set of drivers and performs their movements by using an appropriate road network.

The waste collection in vehicle routing problem (VRP) is due to disposal operations. Vehicles start or end their routes at the empty depot. Empty vehicles leave the depot and collect waste from customers and then emptying themselves at the waste disposal facilities before return to the depot empty. When a vehicle is full, it needs to go to the disposal facilities (transfer station). A vehicle must make a complete trip to make disposal operation per day. Problem arises when a large number of customers exist so that to decision must be made to decide the best solution where a vehicle should make the collection first between the customers before emptying at disposal facilities. The large number of customers makes the waste collection in
VRP becomes complicated because of increasing number of possible solution $(n !)$. If the number of customers is given by $\mathrm{N}$ which is large, therefore we have to solve $\mathrm{N}$ ! of possible solution.

Heuristic algorithm is a procedure that is used to find a good feasible solution that is at least reasonably close to being optimal. The classical algorithm was first proposed by Clarke and Wright (1964) to solve CVRP.Another popularly known heuristic algorithm is tabu search (TS). The best solution at each iteration in the neighborhood of the current solution is selected as the new current solution, even if it leads to an increase in solution cost. It has been used in solving MDVRP with capacity and route length restrictions (Renaud et. al., 1996). TS is used to solve the waste collection VRP problem and to obtain the ordering of the path that produces the shortest distance and the minimum cost. The simplest VRP which only involving only single depot, single disposal facility and the distance between the two customers is Euclidean. It can be calculated using the equation:

$$
d_{i j}=\sqrt{\left(x_{i}-x_{j}\right)^{2}+\left(y_{i}-y_{j}\right)^{2}} \text { for } i, j=0,1,2, \ldots
$$

\section{LITERATURE REVIEW}

The basic Vehicle Routing Problem (VRP) is one of the most widely studied problems in combinatorial optimization. VRP has started over nearly 50 years ago by Dantzig and Ramser (1959) that introduced capacitated VRP (CVRP) by describing a real - world problem 
concerning dispatching gasoline delivery trucks between a bulk of terminal and large numbers of service stations. When the numbers of the service stations become larger, options of routes increase thus makes the finding an improved route to yield an optimal solution become more challenging. Therefore, the capacity of each truck is explicitly considered known as Capacitated VRP (CVRP). Besides that, various classes of traditional VRP have been identified such as Time-Dependent VRP (TDVRP), pickup and Delivery Problem (PDP), Multi-Depot VRP (MDVRP), Location Routing Problem (LRP), Fleet Size and Mix VRP (FSMVRP) and VRP with Time Windows (VRPTW).

The related papers that dealing with the collecting waste during the past year will be briefly review as follow. Kulcar (1996) developed a methodology to illustrate how waste transportation costs can be minimizing in a case study of optimizing solid waste collection in Brussel. Eisenstein and Iyer (1997) solving the residential waste collection problem in the city of Chicago. Meanwhile, De Meulemeester et. al., (1997) dealt with the problem of delivering empty skips and collecting full skips from customers. Chang et al., (1997) analyzed alternative solid waste collection strategies for a city in Taiwan by combining GIS functions with analytical model.

Tung and Pinnoi (2000) presented a tabu search algorithm for the periodic version of the problem where routes must be design over a planning horizon for more than one time period to meet customer requirements. In contrast, Bodin et al. (2000) presented four heuristic algorithms and produced computational results for solving a sanitation routing problem that called the roll-on-roll-off vehicle routing problem. Mourao (2000) used a route firstcluster second approach where a giant tour is generated first, and then decomposed with a lower - bounding method into a set that are feasible. Different from others, Angelilie and Speranza (2002) apply a tabu search algorithm with four move operators to solve a periodic vehicle routing problem with intermediate facilities (PVRP-IF).

Teixeira et. al., (2004) applied a heuristic approach for a PVRP for the separate collection of three types of waste: glass, paper and plastic/metal. Meanwhile, Aringhieri et. al., (2004), describe special waste collected from containers at collection centers instead of each household. The problem can be modeled as the rollonrolloff VRP. Sahoo et. al., (2005) addressed a real life waste collection vehicle routing problem with time windows (VRPTW) with considerable of multiple disposal trips and drivers' lunch breaks. A capacitated clusteringbased waste collection VRPTW algorithm is developed to improve the route compactness and work balancing. Alagoz and Kocasoy (2008) used a commercial vehicle routing package to consider health waste collection.

Nuortio et. al., (2006) described the optimization of vehicle routes and schedules for collecting municipal solid waste in Eastern Finland. The method that has been used to solve the problem is guided variable neighborhood thresholding metaheuristic. Hemmelmayr et. al., (2009) considered a periodic problem by using dynamic programming to sequence disposal facility visits within a variable neighborhood search approach. They designed over a multi-day planning horizon so as to meet customer service requirements. Then, Benjamin and Beasley (2010) produced better quality solutions from Sahoo et. al., (2005) because they solved by using two metaheuristic algorithms which is tabu search and variable neighborhood search that are based around the neighbor sets. Fooladi et. al., (2013) used LINGO 0.9 software to solve a mixed integer nonlinear model in order to find the optimal route for garbage transport vehicles.

\section{TABU SEARCH ALGORITHM}

Glover (1986) introduced Tabu Search (TS) as a metaheuristic superimposed on another heuristic. Tabu generally means socially or culturally proscribed that forbidden to be used, mentioned or approached because of social or cultural rather than legal prohibitions. TS is a neighborhood search method to pursue Local Search whenever it encounters a local minimum by allowing nonimproving moves. TS always used to solve combinatorial optimization problems. It is one of the best methods used at finding solutions close to optimality in large combinatorial problems. Tabu Search algorithm this study:

Step 1 : Choose an initial solution, $i$ in $S$. Set $i^{*}=i$ and $k=0$.

Step 2 : Set $k=k+1$ and generate a subset $G^{*}$ of solution in $N(i, k)$ such that either one of the Tabu condition is violated or at least one of the aspiration conditions holds.

Step 3 : Choose a best $j$ in $G^{*}$ and set $i=j$.

Step 4 : If $f(i)<f\left(i^{*}\right)$ then set $i^{*}=i$.

Step 5 : Update Tabu and Aspiration Conditions.

Step 6 : If a stopping condition is met, then stop. Else, go to step 2.

The formulation of the VRP model as follow by let:

i. The total number of customers, $c_{i, j}$ on the map is represented as $n$.

ii. The length between each customer, $c_{i}$ and $c_{j}$ is represented as $d_{i, j}$.

iii. For each link $c_{i, j}$ is 1 , if $\operatorname{link} c_{i, j}$ is part of the visit. Else is 0 .

iv. The edges $v_{i, j}=0$, indicates no distances between same customer, $c_{i, j}$.

v. The set of arcs of the graph is A.

\subsection{Mathematical Modelling of Vehicle Routing Problem}

Model for the problem $\mathrm{P}$ is known as the cost function:

$$
\text { P: MINIMIZE } \quad C=\sum_{i=0}^{n} \sum_{j=0}^{j=i} c_{i j} d_{i j}
$$


Subject to:

$$
\begin{array}{rlrl}
\sum_{i=1}^{n} c_{i, j} & =1 & & \text { for all } i, j=0,1,2, \ldots, n \\
\sum_{j=1}^{n} c_{i, j} & =1 & & \text { for } \text { all } i, j=0,1,2, \ldots, n \\
\sum_{i, j \in A}^{n} c_{i, j} & \leq(n-1) & & \text { for } \text { all } i, j \in A \\
c_{i, j} & \in\{0,1\} & \text { for } \quad \text { all } i, j=0,1,2, \ldots, n
\end{array}
$$

Equation (2) is the objective function which will minimize the total length. Equation (3) ensures that each customer is visited from only one other customer. Equation (4) ensures that each customer departs to only one other customer. Equation (5) ensures that each visit has no more than $(n-1)$ arcs in the set $n$. Equation (6) is the integrality constraints which:

$$
c_{i j}=\left\{\begin{array}{lc}
1 & \text { if customer } i \text { and } j \text { are connected } \\
0 & \text { if customer } i \text { and } j \text { are not connected }
\end{array}\right.
$$

In solving VRP problem, factors such as the capacity of waste collection for the vehicle will not be considered as the vehicle is very big.
VRP is viewed as the Multi Travelling Salesman Problem (M-TSP). It is belong to the group of NP-hard problems because the time for solving the problem will increase exponentially with increased number of input $n$. in this paper, we consider small example of waste collection vehicle routing problem in this paper.

Let the coordinate of customer 1 is $\left(x_{1}, y_{1}\right)$ and coordinate of customer 2 is $\left(x_{2}, y_{2}\right)$. The Euclidean distance between two customers can be calculated by the equation (1). The objective function in this problem is to minimize the cost function. The cost function of this VRP is the total sum of distance travelled. Then, cost function, $C$ can be written as below:

$$
\text { Minimize } C=\sum_{i=0}^{n} \sum_{j=0}^{j=i} c_{i j} d_{i j}
$$

where $n=$ total number of the customers and

$$
c_{i j}=\left\{\begin{array}{lc}
1 & \text { if customer } i \text { and } j \text { are connected } \\
0 & \text { if customer } i \text { and } j \text { are not connected }
\end{array}\right.
$$

The following Table 1 shows the list of coordinates for a depot and 5 customers.

The distance between Disposal Facility, 0 and customer 1 by applying equation 1 is 45.54 . By the same formula and calculations, the distance for all pair of customers are given in Table 2 below:

\subsection{Experimental Tabu Search: Result}

Table.1 Customer Data

\begin{tabular}{ccc}
\hline Customer Number & $\mathbf{x}$ & $\mathbf{y}$ \\
\hline 0 & 10 & 30 \\
1 & 25 & 73 \\
2 & 48 & 65 \\
3 & 45 & 42 \\
4 & 70 & 45 \\
5 & 40 & 10 \\
\hline
\end{tabular}

Table 2. Distance matrix between the customers

\begin{tabular}{cccccccc}
\hline Customer Number & $\mathbf{0}$ & $\mathbf{1}$ & $\mathbf{2}$ & $\mathbf{3}$ & $\mathbf{4}$ & $\mathbf{5}$ & \\
\hline 0 & $\mathrm{x}$ & 45.54 & 51.66 & 37.00 & 61.85 & 36.06 \\
1 & 45.54 & $\mathrm{x}$ & 24.35 & 36.89 & 53.00 & 64.76 \\
2 & 51.66 & 24.35 & $\mathrm{x}$ & 23.19 & 29.73 & 55.58 \\
3 & 37.00 & 36.89 & 23.19 & $\mathrm{x}$ & 25.18 & 32.39 \\
4 & 61.85 & 53.00 & 29.73 & 25.18 & $\mathrm{x}$ & 46.1 \\
5 & 36.06 & 64.76 & 55.58 & 32.39 & 46.10 & $\mathrm{x}$ \\
\hline
\end{tabular}

A basic TS implementations namely the way the initial solution is generated, the moves, the tabu list size and the stopping criteria used in this study will be discussed. So, the elements of the TS approach are: i. Initial trial solution: 0-1-2-3-4-5-0.

ii. Move: drop two arcs existing and add two new arcs. 
iii. $\quad$ Tabu List size, $\left|T_{S}\right|=2$.

iv. Tabu restriction, $\operatorname{TAB}(i, j)=\left|T_{s}\right|$

$\operatorname{TAB}(i, j)$ denotes the tabu status of the $i^{t h}$ attribute. This can be defined by $\operatorname{TAB}(i, j)=\left|T_{s}\right|$. (i,j) means we begin at node $I$ and end at node $j$. Added arcs are choose randomly from the routes. After that, added arcs will be banned for the next two iterations. That means, we cannot drop these arcs for next two iterations. We will update the tabu status and tabu list size in every iteration.

v. Stopping criteria

Stop after 3 iterations.
From the implementations of TS above, we get initial trial solution which is $C^{0}: 0-1-2-3-4-5-0$. The total distance is $d\left(C^{0}\right): 200.42$ and the aspiration level, $C_{A}$ for $C^{0}$ is 200.42. Serve $C^{0}$ as best move so far.Then find all the necessary move for $C^{0}$. After that, we compare all the total distance based on necessary move for $C^{0}$ (refer on Table 3). Since we are looking for minimum solution, the trial solution we state as $C^{1}=\mathbf{0 - 1 - 2 - 4 - 3 - 5 - 0}$ with a total distance of $\mathbf{1 9 3 . 2 5}$ is the best current solution. So that, the $\operatorname{arcs}(\mathbf{2}, \mathbf{4})$ and $(\mathbf{3 , 5})$ have been banned. That means arcs $(2,4)$ and $(3,5)$ cannot be drop for next two iterations.

Table 3 Necessary move for $C^{0}$

\begin{tabular}{cccr}
\hline Dropped Arc & Added Arc & $\begin{array}{c}\text { Trial Solution Obtained } \\
\text { (route) }\end{array}$ & Total Distance \\
\hline$(0,1),(2,3)$ & $(0,2),(1,3)$ & $0-2-1-3-4-5-0$ & 220.24 \\
$(0,3),(1,4)$ & $(0,1),(3,4)$ & $0-3-2-1-4-5-0$ & 219.70 \\
$(0,4),(1,5)$ & $(0,1),(4,5)$ & $0-4-2-3-1-5-0$ & 252.47 \\
$(0,5),(1,0)$ & $(0,1),(5,0)$ & $0-5-2-3-4-1-0$ & 238.55 \\
$(1,3),(2,4)$ & $(1,2),(3,4)$ & $0-1-3-2-4-5-0$ & 217.51 \\
$(1,4),(2,5)$ & $(1,2),(4,5)$ & $0-1-4-3-2-5-0$ & 238.55 \\
$(1,5),(2,0)$ & $(1,2),(5,0)$ & $0-1-5-3-4-2-0$ & 249.26 \\
$(\mathbf{2}, \mathbf{4}),(\mathbf{3 , 5})$ & $(\mathbf{2 , 3}),(4,5)$ & $\mathbf{0 - 1}-\mathbf{2}-4-\mathbf{3 - 5}-\mathbf{0}$ & $\mathbf{1 9 3 . 2 5}$ \\
$(2,5),(3,0)$ & $(2,3),(5,0)$ & $0-1-2-5-4-3-0$ & 233.75 \\
$(3,5),(4,0)$ & $(3,4),(5,0)$ & $0-1-2-3-5-4-0$ & 233.45 \\
\hline
\end{tabular}

Since the objective function $C^{1}<C^{0}$, the best solution is 193.25. Assign $C^{0} \leftarrow C^{1}$. $C^{1}$ has the best current solution. We update the tabu list, $\left|T_{S}\right|$ and aspiration level, $C_{A}$. The Tabu List, $\left|T_{S}\right|$ for iteration 1 is $\operatorname{TAB}(2,4)=2$ $\operatorname{TAB}(3,5)=2$ while The Aspiration Level, $C_{A}$ is 193.25(refer to Table 4). Then, we repeat the same step for iteration 2 and 3 and get Table 5 and 6 as below:

Table.4 The Tabu List, $\left|T_{S}\right|$ and The Aspiration Level, $C_{A}$ for iteration 1

\begin{tabular}{cc}
\hline The Tabu List, $\left|T_{S}\right|$ & The Aspiration Level, $C_{A}$. \\
\hline $\operatorname{TAB}(2,4)=2$ & 193.25 \\
$\operatorname{TAB}(3,5)=2$ & \\
\hline
\end{tabular}

Table 5 The Tabu List, $\left|T_{S}\right|$ and The Aspiration Level, $C_{A}$ for iteration 2

\begin{tabular}{cc}
\hline The Tabu List, $\left|T_{S}\right|$ & The Aspiration Level, $C_{A}$. \\
\hline $\operatorname{TAB}(2,4)=1$ & 193.25 \\
$\operatorname{TAB}(3,5)=1$ & \\
$\operatorname{TAB}(4,5)=2$ &
\end{tabular}

$\operatorname{TAB}(3,0)=2$

Table.6 The Tabu List, $\left|T_{S}\right|$ and The Aspiration Level, $C_{A}$ for iteration 3

\begin{tabular}{cc}
\hline The Tabu List, $\left|T_{S}\right|$ & The Aspiration Level, $C_{A}$. \\
\hline $\operatorname{TAB}(4,5)=1$ & 193.25 \\
$\operatorname{TAB}(3,0)=1$ & \\
$\operatorname{TAB}(2,3)=2$ & \\
$\operatorname{TAB}(4,0)=2$ & \\
\hline
\end{tabular}

Therefore, we note that our aspiration level is still 193.25, so that route $\mathbf{0 - 1 - 2 - 4 - 3 - 5 - 0}$ is accepted as the optimal solution for this problem.

\section{CONCLUSION}

As a conclusion, tabu search algorithm is used to solve small example of vehicle routing problem involving 5 customers and a disposal facility which also known as depot. Every iteration consist of 5 steps that to be fulfilled in order to find the minimum cost function based on 
minimum total distanced travelled by the vehicles. The tabu search approach finally give the result that route 0-1-2-4-3-

5-0 is accepted as the optimal solution for this problem. This means that minimum cost function which is 193.25 can be obtained when vehicles collecting waste collection start from disposal facility then go to customer 1, customer 2, customer 4 , customer 3 and customer 5 before going back to the disposal facility again. To further research, we solve the waste collection vehicle routing problem for larger data set.

\section{REFERENCES}

[12] Alagoz, A.Z.; Kocasoy, G. Care Waste Collection and Transportation in Istanbul. 28 (2008) 1461-1471.

[13] Angelelli, E.; Speranza, M.G. European journal of Operational Rresearch. 137 (2002) 233-247.

[14] Aringhieri, R.; Bruglieri, M.; Malucelli, F.; Nonato, M. A particular vehicle routing problem arising in the collection and disposal of special waste. Presented at Tristan 2004, Guadeloupe, French West Indies.

[15] Benjamin, A.M.; Beasley, J.E. Journal of Computer and Operations Research. 37 (2010) 2270-2280.

[16] Bodin, L.; Mingozzi, A.; Baldacci, R.; Ball, M. Journal of Transportation science. 34 (2000) 271-288.

[17] Chang, N.-B.; Lu, H. Y.; Wei, Y. L. Journal of Environmental Engineering. 123 (1997) 901-910.

[18] Clarke, G.; Wright, J.W. Journal of Operations Research. 12 (1964) 568-581.

[19] Dantzig, G. B.; Ramser, J. H. Journal of Management Science. 6 (1959) 80-91.

[20] De Meulemeester, L.; Laporte, G.; Louveaux, F.V.; Semet, F. Journal of the Operational Research Society. 48 (1997) 57-64.

[21] Eisenstein, D. D.; Iyer. A. V. Journal of Management science. 43 (1997) 922-933.
[1] Fooladi, S.; Fazlollahtabar H.; Mahdari I. International Journal of Applied Operational Research. 3 (2013) 105-111.

[2] Glover, F. Journal of computational operations research. 13 (1986) 533-549.

[3] Hemmelmayr, V.; Doerner, K.F.; Hartl, R.F.; Rath, S. Metaheuristic for a real world solid waste collection problem. Working paper available from the third author of the Department of Business Administration, University of Vienna, Austria. (2009).

[4] Kulcar, T. European Journal of Operational Research. 90 (1996) 71-77.

[5] Laporte, G. The Vehicle Routing Problem: An overview of exact and approximate algorithms. (1991).

[6] Mourou, M. European journal of Operational Research. 121 (2000) 420-434.

[7] Nuortio, T.; Kytojoki, J.; Niska, H.; Braysy, O. Journal of Expert system with Application. 30 (2006) 223-232.

[8] Renaud J.; Laporte G.; Boctor F. F. A Tabu Search Heuristic for the Multi Depot Vehicle Routing Problem, vol 23, no 3, 1996, pp. 229-235.

[9] Sahoo, S.; Kim,S.; Kim, B.I.; Kraas, B.; Popov, A. Interfaces 35 (2005) 24-36.

[10] Teixera, J.; Antunes, A.P.; Sousa, J.P. European Journal of Operational Research. 58 (2004) 543-554.

[11] Tung, D. V.; Pinnoi, A. European Journal of Operational Research. 125 (2000) 449-468. 